\title{
The Flexion Initiation Test and an Evidence-Based Diagnostic Algorithm for Distal Biceps Tendon Tears
}

\author{
Olivia J. Bono, B.A., Sarav S. Shah, M.D., Justin Peterson, D.O., \\ Samuel W. Golenbock, Ph.D., and Glen Ross, M.D.
}

\begin{abstract}
Purpose: The purpose of this study was (1) to assess the flexion initiation test's (FIT) ability to detect distal biceps tendon tears (DBTT) in a cohort of consecutive patients presenting with elbow pain and (2) to generate a reliable evidence-based diagnostic algorithm using a combination of both the FIT and hook tests. Methods: We performed a retrospective review of 125 consecutive patients who presented with elbow pain, all of which had the FIT and hook test performed prior to imaging/further intervention. The integrity of the tendon was determined during surgery or by magnetic resonance imaging. Sensitivity, specificity, positive predictive value, and negative predictive value were determined for the FIT and hook test. Results: Our evidence-based diagnostic algorithm showed that when both test results are in agreement, there is a $100 \%$ diagnostic accuracy for detecting what prior authors have termed surgically indicated tears (complete ruptures and high-grade partial tears) and biceps pathology that can be treated with nonoperative management. The FIT demonstrated $100 \%$ sensitivity for surgically indicated tears. The hook test demonstrated $100 \%$ sensitivity for complete ruptures, but $18 \%$ sensitivity for diagnosing partial tears. Conclusions: The FIT, which is aimed at improving diagnostic acuity of high-grade partial thickness tears, demonstrated a 93\% sensitivity and $96 \%$ specificity overall and a $100 \%$ sensitivity for complete ruptures and high-grade partial tears. The evidence-based diagnostic algorithm using the combination of the FIT and hook test demonstrates high accuracy for the diagnosis of both complete and high-grade partial DBTTs. The methodology may help to prevent diagnosis delays, improve patient education, and preserve the option for timely primary surgical repair in the treatment of DBTTs. Level of Evidence: Level IV, diagnostic.
\end{abstract}

$\mathbf{T}$ here has been a growing incidence of distal biceps tendon tears (DBTTs), reported at around 2.55 per 100,000 patient-years. ${ }^{1}$ Typically seen in middle-aged men, the injury mechanism occurs when a sudden eccentric load is applied as the arm is brought from flexion into extension. ${ }^{2}$ Patients presenting acutely display pain, ecchymosis, and weakness of flexion/supination making activities that require strength in

From the Department of Orthopedic Surgery, New England Baptist Hospital, Boston, Massachusetts, U.S.A. (O.J.B., S.S.S., G.R.); Department of Research, New England Baptist Hospital, Boston, Massachusetts, U.S.A. (S.W.G.); and Aspen Orthopedic Specialists, Brookfield, Wisconsin, U.S.A. (J.P.)

The authors report that they have no conflicts of interest in the authorship and publication of this article. Full ICMJE author disclosure forms are available for this article online, as supplementary material.

Received October 3, 2020; accepted January 20, 2021.

Address correspondence to Glen Ross, M.D., New England Baptist Hospital, Pro Sports Orthopedics, 20 Guest Street, Suite 225, Brighton, MA 02135 U.S.A.E-mail:ross2ortho@aol.com

(C) 2021 THE AUTHORS. Published by Elsevier Inc. on behalf of the Arthroscopy Association of North America. This is an open access article under the CC BY-NC-ND license (http://creativecommons.org/licenses/by-nc-nd/4.0/). 2666-061X/201642

https://doi.org/10.1016/j.asmr.2021.01.010 supination, such as opening a jar, difficult for patients to perform. ${ }^{3}$ Primarily responsible for supination of the forearm, the biceps brachii muscle is also important in elbow flexion along with the brachioradialis and brachialis. $^{4-6}$ The bicipital aponeurosis (lacertus fibrosus) arises at the junction of the musculotendinous unit and the distal biceps tendon, inserting on the subcutaneous border of the ulna, and has been discussed to have a role in force transmission during flexion. ${ }^{7,8}$ When compared with the contralateral arm, complete rupture of the distal biceps can produce a $40 \%$ loss of supination strength, a $47 \%$ loss of supination endurance, and a $21 \%$ to $30 \%$ loss of flexion strength at the elbow. ${ }^{5,9}$

Timely diagnosis of a complete distal biceps tendon rupture enhances the ability to execute a primary repair and to reestablish motion and strength. ${ }^{7}$ Physical examination and clinical tests are the hallmark of diagnosing distal biceps tendon ruptures. Magnetic resonance imaging (MRI) is rarely necessary for diagnosis of a complete tear, although it has utility in distinguishing between complete versus partial tear, muscle substance versus tendon tear, and degree of retraction for surgical planning. ${ }^{1}$ However, in the acute setting, MRI may cause a delay in patient's treatment 
and may add cost to the patient and medical system. ${ }^{10}$ Partial-thickness tears are less common, likely underdiagnosed, and high-grade partial thickness tears place patients at risk for rupture completion. ${ }^{10}$

Historically, physical examination tests, such as the hook test, ${ }^{11}$ have focused on examination with the elbow at $90^{\circ}$ of flexion. However, the biceps achieves maximal voluntary contraction at $7.3^{\circ}$ elbow flexion. ${ }^{12}$ Falsenegative examination may be elicited when investigating the integrity of the muscle or upper arm contour as the elbow flexes if the lacertus fibrosus remains intact in the presence of a complete rupture of the distal biceps tendon. ${ }^{2,13}$ Furthermore, the hook test has deficiencies in diagnosing partial tears because the maneuver will falsely demonstrate an intact tendon. ${ }^{10}$ Thus there is a need for improved physical examination tools to prevent diagnosis delays, improve patient education, and preserve the option for primary surgical repair in the treatment of DBTTs. The flexion initiation test (FIT) was developed as an adjunct diagnostic tool during the physical examination to isolate the biceps brachii at maximal contraction and more accurately assess the integrity of the tendon aimed at improving diagnostic acuity of high-grade partial thickness tears. ${ }^{14,15}$ The study's objectives are (1) to assess the FIT's ability to detect DBTTs in a cohort of consecutive patients presenting with elbow pain and (2) to generate a reliable evidence-based diagnostic algorithm using a combination of both the FIT and hook tests. We hypothesize that the FIT, when used in combination with the hook test, will improve diagnostic accuracy for detecting DBTTs, enhancing clinical decision-making and patient education.

\section{Methods}

In this IRB approved study, a retrospective chart review of prospectively collected data was performed for this observational cross-sectional study. One hundred twenty-five consecutive patients who presented to the senior author with a complaint of elbow pain, either acute $(\leq 4$ weeks between the injury and first clinic visit) or chronic ( $>4$ weeks), ${ }^{10,16}$ between April 1, 2017, and April 1, 2020, were included. Patients were excluded if they (1) were younger than 18 years, (2) had a fracture or dislocation, (3) had previous distal biceps surgery, (4) did not have documented results of both physical exam tests (FIT and hook), or (5) did not have confirmation by MRI or surgery of the integrity of the distal biceps tendon. MRI evaluation was performed by an independent musculoskeletal radiologist. Patient history was collected from the medical record, including patient age, sex, arm dominance, injured arm, and the chronicity of the injury.

A single examiner (G.R.), a fellowship-trained orthopaedic surgeon specializing in shoulder and elbow disorders with over 30 years of experience, performed physical examination testing for all patients. The injured upper extremity was inspected for gross deformity along with ecchymosis of the biceps tendon and muscle belly. The elbow was evaluated for any range of motion deficit in flexion, extension, pronation, and supination. Physical examination maneuvers specific for biceps tendon evaluation were the hook test and the FIT. The physical examination was performed before MRI, if an MRI was ordered, or before viewing the MRI scans if the patient had imaging before the visit.

The primary outcome measure was tear of the distal biceps tendon as confirmed by MRI or intraoperative inspection. Patients were diagnosed with a complete tear if the distal biceps tendon had no intact attachment to the radial tuberosity. A partial tear was defined by the presence of any remaining tendinous attachment to the radial tuberosity, as confirmed by MRI, and surgery, for those who went on to have surgical repair. A highgrade partial tear was defined as greater than $50 \%$ of tendon involvement, whereas a low-grade tear was defined as $<50 \%$ of tendon involvement. ${ }^{17}$ A surgically indicated distal biceps tear was defined as a complete tear or a symptomatic high-grade partial tear. ${ }^{17}$

\section{Statistical Analysis}

The definitive status of the distal biceps tendon was compared against the results of the physical exam tests. Sensitivity, specificity, positive predictive value (PPV), and negative predictive value (NPV) of both the FIT and the hook test were calculated. Accuracy of the two tests combined was also calculated as part of the diagnostic algorithm.

\section{Test Description}

To perform the FIT, the patient is seated with their injured arm fully extended and maximally supinated. The examiner places one hand over the palmar surface of the wrist, and the other hand just proximal to olecranon process (Fig 1). The patient is asked to flex the elbow against the examiner's counter force applied at the wrist. Using a handheld dynamometer, 10 to 15 pounds of resistance was applied. In an electromyographic study, the biceps in forearm supination achieved its peak of over $150 \%$ maximal voluntary contraction at $7.3^{\circ}$ elbow flexion. ${ }^{12}$ Using this information, the FIT was formulated. A positive FIT test result is when the patient cannot overcome the examiner's counter force to initiate the first $10^{\circ}$ to $15^{\circ}$ of elbow flexion. A negative FIT occurs when the patient is able to overcome the examiner's resistance to elbow flexion (Fig 2).

The hook test, initially described by O'Driscoll et al., ${ }^{11}$ is positive when there is an absence of a cord-like structure spanning the antecubital fossa behind which to hook the examiner's finger. A negative hook test result occurs when the examiner is able to hook a finger around the biceps tendon from the lateral side. 


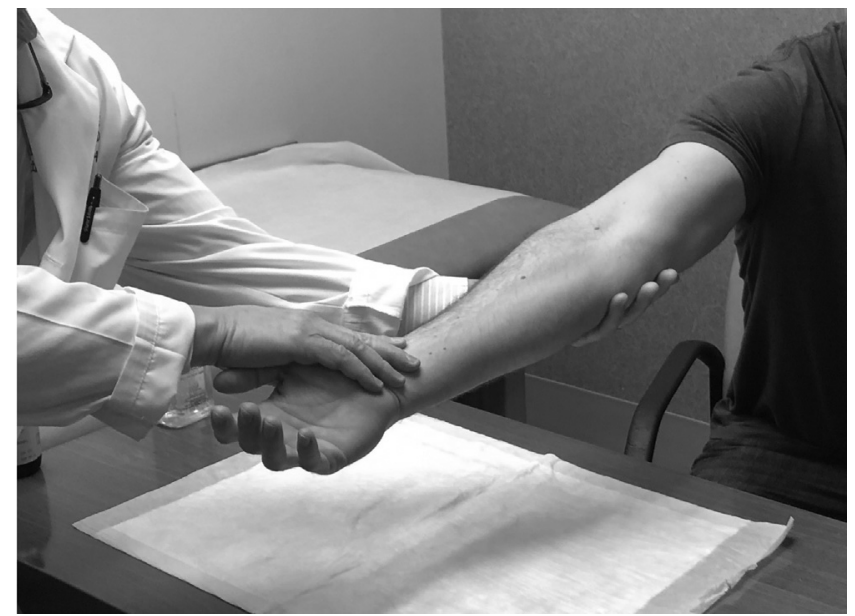

Fig 1. Performing the flexion initiation test (FIT). The patient's arm is placed in full extension and supination. The examiner asks the patient to flex their elbow against resistance placed on the palmar wrist. A positive FIT is when the patient cannot initiate the first $10^{\circ}$ to $15^{\circ}$ degrees of elbow flexion.

\section{Results}

One hundred twenty-five consecutive patients met the inclusion criteria. Twenty-nine patients were confirmed to have DBTTs. Ninety-six patients were confirmed to have intact distal biceps tendons. As displayed in Table 1 , there were 93 males $(74 \%)$ and 32 females $(26 \%)$. Of the 29 patients with confirmed biceps tears, 28 were male $(97 \%)$. The average age of the 125 patients was $53 \pm 11.7$ years. Arm dominance was documented in 104 of 125 patients $(83 \%)$, with the dominant extremity involved in 67 of 104 patients $(64 \%)$. Twenty-three patients had an acute injury $(\leq 4$ weeks between the injury and first clinic visit), whereas 102 had a chronic injury (>4 weeks). Of the 29 biceps tear patients, $17(59 \%)$ presented with an acute injury.

Validation of the FIT and hook test was performed via intraoperative diagnosis or MRI. If the patient did not undergo distal biceps tendon repair, the status of the tendon was confirmed by MRI. The integrity of the distal biceps tendon was confirmed by surgery alone in 7 patients $(6 \%)$, MRI alone in 101 patients $(80 \%)$, and by both MRI and surgery in 17 patients (14\%) (Table 1). There were 18 patients with complete DBTTs and 11 patients with partial tears. Of the 11 patients with partial tears, 6 were confirmed to have a highgrade partial tear by both MRI and surgery, and 5 patients had MRI-confirmed low-grade partial tears. Fifteen patients showed tendinopathy or tendinosis of the distal biceps tendon on MRI and were not considered to have a tear.

The FIT had a sensitivity of $93 \%$ and a specificity of $96 \%$ overall (Table 2), and a PPV and NPV of 0.87 and 0.98 , respectively. When looking at both complete tendon ruptures and high-grade partial tears, the FIT had a sensitivity of $100 \%$.
By comparison, the hook test had a sensitivity of $69 \%$ and a specificity of $100 \%$ overall (Table 2), and a PPV and NPV of 1.00 and 0.91 , respectively. Although the hook test had a sensitivity of $100 \%$ for complete tears, it had a sensitivity of only 18\% for partial tears (Table 2 ).

\section{Discussion}

The evidence-based diagnostic algorithm (Fig 3) using the combination of the FIT and hook test discussed in this study demonstrates 100\% accuracy for the diagnosis of what prior authors have described as surgically indicated tears (complete and high-grade partial DBTTs). ${ }^{17}$ The algorithm may help to prevent diagnosis delays, improve patient education, and preserve the option for primary surgical repair in the treatment of DBTTs. Based on the results of this study, the FIT test can help reliably diagnose both complete and partial DBTTs. With high sensitivity and specificity, the FIT test offers clinicians a straightforward physical examination maneuver that may allow potential avoidance of misdiagnosis or the added cost of advanced imaging. Timely diagnosis of the injury is critical, so that the possibility of surgical management at the time of presentation is not compromised. Chronic ruptures ( $>4$ weeks) often present with proximal retraction of the biceps muscles and scarring to the brachialis, which can make anatomic repair a challenge, ${ }^{7,18}$ potentially requiring excessive elbow flexion or allograft augmentation.

There have been a variety of physical examination tests developed; however, each has constraints. In comparison to the supination resistance test, the FIT is performed in a more anatomically accurate position (biceps in forearm supination achieves its peak contraction at 7.3 degrees of elbow flexion), ${ }^{12}$ versus the $90^{\circ}$ of flexion at which the supination resistance test occurs. ${ }^{19,20}$ Furthermore, there have been no sensitivity/specificity analyses published for the supination resistance test. ${ }^{19-21} \mathrm{O}^{\prime}$ Driscoll et al. ${ }^{11}$ described

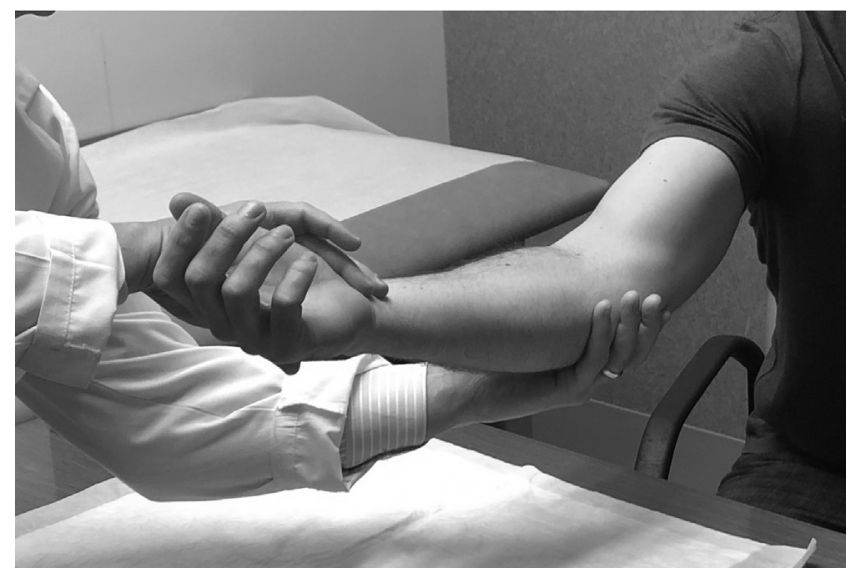

Fig 2. Negative flexion initiation test result. The patient has overcome the examiner resistance to elbow flexion, indicating that the distal biceps tendon remains intact. 
Table 1. Patient Characteristics

\begin{tabular}{|c|c|c|c|}
\hline $\begin{array}{r}\text { Characteristic } \\
\end{array}$ & Total $(\mathrm{n}=125)$ & Biceps Tears $(\mathrm{n}=29)$ & Intact Biceps $(\mathrm{n}=96)$ \\
\hline Age, yr, mean \pm SD (range) & $53.0 \pm 11.7(21-77)$ & $52.0 \pm 12.2(23-76)$ & $54.0 \pm 11.6(21-77)$ \\
\hline \multicolumn{4}{|l|}{ Sex, no. (range) } \\
\hline Male & $93(74)$ & $28(97)$ & $65(68)$ \\
\hline Dominant arm injured, no. (range) & $67(64)$ & $12(48)$ & $55(70)$ \\
\hline \multicolumn{4}{|l|}{ Time since injury, no. (range) } \\
\hline Acute ( $\leq 4$ weeks) & $23(18)$ & $17(59)$ & $6(6)$ \\
\hline Surgery & $7(6)$ & $7(25)$ & $0(0)$ \\
\hline MRI & $101(80)$ & $17(59)$ & $0(0)$ \\
\hline Surgery and MRI & $17(14)$ & $17(59)$ & $0(0)$ \\
\hline
\end{tabular}

*All values given as number of patients (\% of total), except for age.

the hook test for distal biceps tendon ruptures with $100 \%$ sensitivity and $100 \%$ specificity for complete ruptures. However, some authors have found it clinically difficult to reproduce such "degree of certainty." ${ }^{10}$ Additionally, others have reported that this examination can be challenging to administer because it necessitates palpation of the tendon, which is often painful for the patient with an acute injury. ${ }^{22}$

Use of more than 1 physical examination maneuver can improve a physician's ability to diagnose in the acute and chronic settings. Devereaux et al. ${ }^{14}$ demonstrated a $100 \%$ sensitivity and specificity for diagnosing specifically complete ruptures when using a combination of three tests with an accurate patient history, highlighting the importance of using multiple tests to make the correct diagnosis. The diagnostic algorithm described in this study, using both the hook test and FIT, allows reliable evaluation not only for complete tears but also partial tears without MRI. The FIT's superior ability to detect surgically indicated partial tears is apparent when noting the FIT had a sensitivity of $100 \%$ for diagnosing high-grade partial tears. However, because there were only 6 high-grade partial tears, sensitivity calculations in this group warrant further study in a larger population. Under the diagnostic algorithm (Fig 3), when both tests were positive, there was a $100 \%$ accuracy for detecting a surgically indicated DBTT (complete tear or a high-grade partial tear). ${ }^{17}$ Thus the patient can confidently be indicated for surgical repair of the tendon. If both test results are negative, there is high clinical confidence for an intact biceps tendon or one that does not require surgery, given its $100 \%$ accuracy for detecting a distal biceps tendon that is either intact, has a low-grade partial tear, or has tendinosis/tendinopathy, all of which can proceed to nonoperative treatment. In only 11 of 125 patients, the results of the 2 tests were not in agreement (FIT positive, hook test negative). In this scenario, there was an $82 \%$ accuracy for detecting a partial tear or other biceps pathology; therefore we recommend proceeding with confirmatory MRI for clarification on the severity of the pathology. By limiting MRI use to only when the results of the 2 tests are not in agreement, our algorithm allows for more prudent use of resources in a cost-conscious healthcare environment. Because the typical presentation of DBTT is without concomitant pathology, ${ }^{3,23}$ we believe MRI is unnecessary when both the FIT and hook test results are positive, especially given their high specificities $(96 \%$ and $100 \%$, respectively). Our results suggest that the FIT and hook test combined provide sufficient diagnostic accuracy to reliably detect DBTTs and enable clinicians to confidently educate patients on the most efficient and costeffective treatment approach.

The data for the patients included in this study are largely based on MRI, which is an imperfect tool. MRI may report false-negative readings, particularly for lowgrade partial tears ${ }^{24}$; however, our study aimed at identifying a treatment algorithm to safely and accurately diagnose surgically indicated DBTTs, which include complete and high-grade partial tears. ${ }^{17}$ Modern imaging including specific views such as the flexed elbow, abducted shoulder, supinated forearm view may aid in diagnosis of these partial tears. ${ }^{25,26}$ Although MRI

Table 2. Sensitivity and Specificity for Detecting Distal Biceps Tendon Tears Overall, and for Complete Tears and Partial Tears

\begin{tabular}{|c|c|c|c|c|c|}
\hline Specialty Testing & \multicolumn{3}{|c|}{ All Patients $(n=125)$} & $\frac{\text { Complete Tears }(\mathrm{n}=18)}{\text { Sensitivity }}$ & $\frac{\text { Partial Tears }(\mathrm{n}=11)}{\text { Sensitivity }}$ \\
\hline FIT & $93 \%$ & $96 \%$ & $\begin{array}{l}2 \text { False-negatives } \\
4 \text { False-positives }\end{array}$ & $100 \%$ & $82 \%$ \\
\hline Hook & $69 \%$ & $100 \%$ & 9 False-negatives & $100 \%$ & $18 \%$ \\
\hline
\end{tabular}




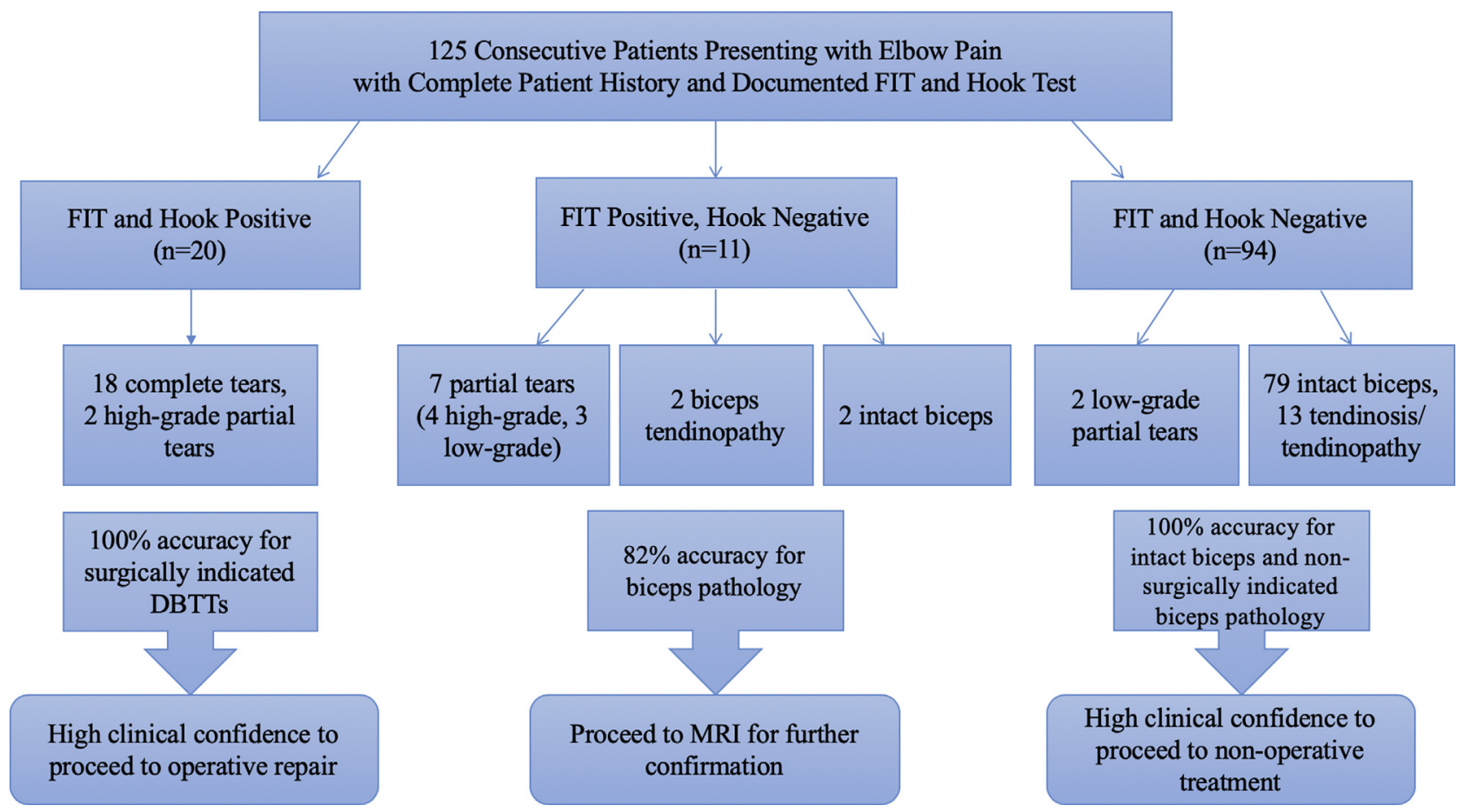

Fig 3. Evidence-based diagnostic algorithm using the flexion initiation test (FIT) and hook test results to safely and accurately diagnose distal biceps tendon tears (DBTTs).

has limitations, studies have shown it has a relatively high sensitivity and specificity, especially for complete tears, and it is more accurate than ultrasound in detecting a tear. ${ }^{24,27}$ Festa et al. ${ }^{24}$ looked at MRI accuracy in diagnosing distal biceps ruptures and found that MRI has $100 \%$ sensitivity and $82 \%$ specificity for complete ruptures. Based on the results of our study, patients with high-grade partial tears may not gain extra benefit from the additional cost of an MRI. Notably, our study may allow for a smaller cohort of patients that would require MRIs, because these advanced imaging tests are usually only ordered in instances of unclear physical examination. Although MRI for distal biceps ruptures still has a role in specific clinical settings, proper clinical evaluation and clinical discretion should be used.

\section{Limitations}

Limitations of this study include that it is a retrospective study with potential selection bias because not all patients were evaluated with the same modalities. Although there is a potential for bias in the interpretation of the FIT, this is minimized by the examiner performing the examination maneuvers on the first clinical visit and after obtaining only a brief history. Also, without further study, the generalizability of the FIT is unproven because the novel physical examination test was evaluated by a single examiner. However, it was developed as an adjunct diagnostic tool during the physical examination, and it is relatively straightforward. Furthermore, although the chronicity of the injury may affect the examiner's ability to perform the FIT, the generalizability of the examination does not seem to be hindered by chronicity of injury; our results show 12 $(41 \%)$ patients with biceps tears had a chronic injury (Table 1), and yet the FIT had only 2 false-negative results in total. Although there was a high sensitivity and specificity, the FIT's 2 false-negative results were for low-grade partial tears, which suggests that a patient may be able to compensate either through their brachialis or remaining biceps tendon. Also, there were 4 false-positive results, which may be related to patient pain tolerance levels. Thus the authors do not recommend using the FIT alone, but as a part of the diagnostic algorithm that can reliably predict when to proceed with operative intervention, conservative management, or obtain advanced imaging.

\section{Conclusions}

The FIT, which is aimed at improving diagnostic acuity of high-grade partial thickness tears, demonstrated a $93 \%$ sensitivity and $96 \%$ specificity overall and a $100 \%$ sensitivity for complete ruptures and highgrade partial tears. The evidence-based diagnostic algorithm using the combination of the FIT and hook test demonstrates high accuracy for the diagnosis of both complete and high-grade partial DBTTs. The methodology may help to prevent diagnosis delays, improve patient education, and preserve the option for timely primary surgical repair in the treatment of DBTTs. 


\section{References}

1. Hsu DAP, Chang KV. Biceps Tendon Rupture. StatPearls Publishing. Updated April 20, 2020, https://www.ncbi. nlm.nih.gov/books/NBK513235/.

2. Sutton KM, Dodds SD, Ahmad CS, Sethi PM. Surgical treatment of distal biceps rupture. J Am Acad Orthop Surg 2010;18:139-148.

3. Frank RM, Cotter EJ, Strauss EJ, Jazrawi LM, Romeo AA. Management of biceps tendon pathology: From the glenoid to the radial tuberosity. J Am Acad Orthop Surg 2018;26:e77-e89.

4. An KN, Hui FC, Morrey BF, Linscheid RL, Chao EY. Muscles across the elbow joint: a biomechanical analysis. J Biomech 1981;14:659-669.

5. Morrey BF, Askew LJ, An KN, Dobyns JH. Rupture of the distal tendon of the biceps brachii. A biomechanical study. J Bone Joint Surg Am 1985;67:418-421.

6. Ruland RT, Dunbar RP, Bowen JD. The biceps squeeze test for diagnosis of distal biceps tendon ruptures. Clin Orthop Relat Res 2005;437:128-131.

7. Ramsey ML. Distal biceps tendon injuries: diagnosis and management. J Am Acad Orthop Surg 1999;7:199-207.

8. Snoeck O, Lefevre P, Sprio E, et al. The lacertus fibrosus of the biceps brachii muscle: An anatomical study. Surg Radiol Anat 2014;36:713-719.

9. Safran MR, Graham SM. Distal biceps tendon ruptures: Incidence, demographics, and the effect of smoking. Clin Orthop Relat Res 2002:275-283.

10. Alentorn-Geli E, Assenmacher AT, Sanchez-Sotelo J. Distal biceps tendon injuries: A clinically relevant current concepts review. EFORT Open Rev 2016;1:316-324.

11. O'Driscoll SW, Goncalves LB, Dietz P. The hook test for distal biceps tendon avulsion. Am J Sports Med 2007;35: 1865-1869.

12. Caufriez B, Dugailly PM, Brassinne E, Schuind F. The role of the muscle brachioradialis in elbow flexion: An electromyographic study. J Hand Surg Asian Pac 2018;23: 102-110.

13. Wylie JDWM, DeVenere J, Mazzocca AD. Distal biceps tendon injuries: Treatment of partial and complete tears. Op Tech Sports Med 2017;25:304-309.

14. Devereaux MW, ElMaraghy AW. Improving the rapid and reliable diagnosis of complete distal biceps tendon rupture: a nuanced approach to the clinical examination. Am J Sports Med 2013;41:1998-2004.

15. Taylor SA, Newman AM, Dawson C, et al. The "3-pack" examination is critical for comprehensive evaluation of the biceps-labrum complex and the bicipital tunnel: A prospective study. Arthroscopy 2017;33:28-38.

16. Grewal R, Athwal GS, MacDermid JC, et al. Single versus double-incision technique for the repair of acute distal biceps tendon ruptures: a randomized clinical trial. J Bone Joint Surg Am 2012;94:1166-1174.

17. Bain GI, Johnson LJ, Turner PC. Treatment of partial distal biceps tendon tears. Sports Med Arthrosc Rev 2008;16: 154-161.

18. Leighton MM, Bush-Joseph CA, Bach BR Jr. Distal biceps brachii repair. Results in dominant and nondominant extremities. Clin Orthop Relat Res 1995;317:114-121.

19. Bain GI, Durrant AW. Sports-related injuries of the biceps and triceps. Clin Sports Med 2010;29:555-576.

20. Kane SF, Lynch JH, Taylor JC. Evaluation of elbow pain in adults. Am Fam Physician 2014;89:649-657.

21. Kelly EW, Morrey BF, O'Driscoll SW. Complications of repair of the distal biceps tendon with the modified twoincision technique. J Bone Joint Surg Am 2000;82: 1575-1581.

22. Metzman LS, Tivener KA. The supination-pronation test for distal biceps tendon rupture. Am J Orthop (Belle Mead NJ) 2015;44:E361-E364.

23. McDonald LS, Dewing CB, Shupe PG, Provencher MT. Disorders of the proximal and distal aspects of the biceps muscle. J Bone Joint Surg Am 2013;95:1235-1245.

24. Festa A, Mulieri PJ, Newman JS, Spitz DJ, Leslie BM. Effectiveness of magnetic resonance imaging in detecting partial and complete distal biceps tendon rupture. J Hand Surg Am 2010;35:77-83.

25. Giuffre BM, Moss MJ. Optimal positioning for MRI of the distal biceps brachii tendon: flexed abducted supinated view. AJR Am J Roentgenol 2004;182:944-946.

26. Fitzpatrick D, Menashe L. Magnetic resonance imaging evaluation of the distal biceps tendon. Am J Orthop (Belle Mead NJ) 2018;47(5). doi:10.12788/ajo.2018.0037.

27. Lynch J, Yu CC, Chen C, Muh S. Magnetic resonance imaging versus ultrasound in diagnosis of distal biceps tendon avulsion. Orthop Traumatol Surg Res 2019;105:861-866. 